\title{
Low-Power Electro-Optic Actuators for Large-Scale Programmable Photonic Circuits
}

\author{
Wim Bogaerts ${ }^{a, b}$, Lukas Van Iseghem ${ }^{a, b}$, Pierre Edinger ${ }^{c}$, Hamed Sattari $^{d}$, \\ Alain Yuji Takabayashid ${ }^{d}$ Xiangfeng Chen ${ }^{\mathrm{a}, \mathrm{b}}$, Hong Deng $^{\mathrm{a}, \mathrm{b}}$, Peter Verheyen ${ }^{\mathrm{e}}$, \\ Antonio Ribeiro $^{\text {a,b }}$, Umar Khan ${ }^{a, b}$, Niels Quack ${ }^{d}$, Kristinn B. Gylfason ${ }^{c}$ \\ ${ }^{a}$ Ghent University - IMEC, Photonics Research Group, Department of Information Technology, Belgium \\ ${ }^{b}$ Center for Nano- and Biophotonics, Ghent University, Belgium \\ ${ }^{c}$ KTH Royal Institute of Technology, SE-100 44 Stockholm, Sweden \\ ${ }^{d}$ École Polytechnique Fédérale de Lausanne (EPFL), 1015 Lausanne, Switzerland \\ ${ }^{e}$ imec vzw. 3DSIP Department, Si Photonics Group, Kapeldreef 75, 3001 Leuven, Belgium \\ wim.bogaerts@ugent.be
}

\begin{abstract}
Photonic integrated circuits are becoming increasingly more complex, especially with the emergence of programmable photonic circuits. These require many tunable photonic elements, such as electro-optic phase shifters and tunable couplers. We will discuss our progress in compact, low-power silicon photonics actuators based on heaters, liquid crystal and MEMS that can be scaled up to large circuits. (C) 2021 The Author(s)
\end{abstract}

\section{Introduction}

Silicon photonics, with its high refractive index contrast and industrial-scale fabrication technology, allows for increasingly large photonic circuits, integrating thousands of functional elements on a chip. Such large circuits are impacted by fabrication variations, and we see a growing use of active tuners to compensate for those imperfections. This trend is most pronounced in the emerging programmable photonic circuits, where the flow of light is dynamically controlled in a waveguide mesh connected by tunable couplers and phase shifters [1]. In such meshes, the electro-optic actuators for the phase shifts and coupling must have a short optical length, a low optical insertion loss, and low electrical power consumption. The key actuation mechanism is to induce an optical phase shift (without introducing loss). The most common approach today is through the use of heaters [2], but this can be power hungry. In silicon we also see carrier-induced phase modulation for high-speed operation, but this effect also introduces significant optical loss. It is possible to introduce electro-optic materials near the silicon waveguide to tune the phase shift. One such example is liquid crystals [3] which has an extremely high optical birefrigence that can be electrically controlled. Alternatively, one can also move the silicon itself, using microelectromechanical systems (MEMS) [4]. Some of these mechanisms are illustrated in Fig. 1]. When developing a new phase shifter mechanism, it is essential to integrate it with the other functions in a silicon photonics platform, such as low-loss waveguides, high-speed modulators or photodetectors. In this paper, we will present our recent progress in implementing low-power phase shifters using heaters, liquid crystals and MEMS.

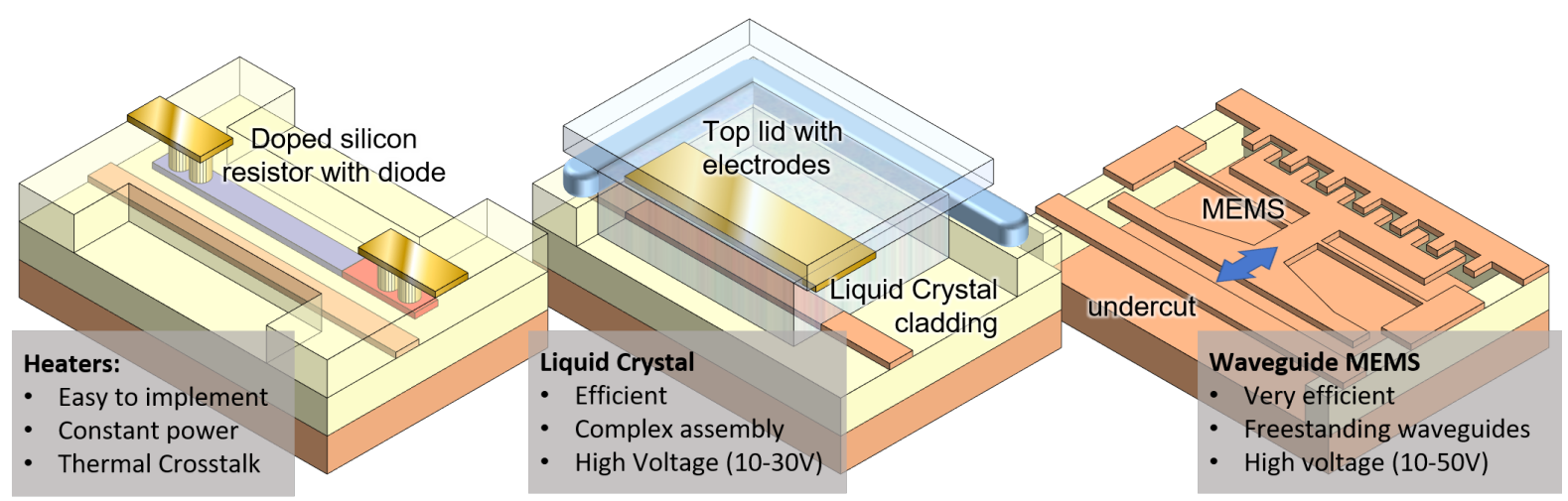

Fig. 1. Different actuation mechanisms for electro-optic phase shifters in silicon photonic circuits. Left to right: heaters (which can have an integrated diode) [5], liquid crystals [3], MEMS [4] 


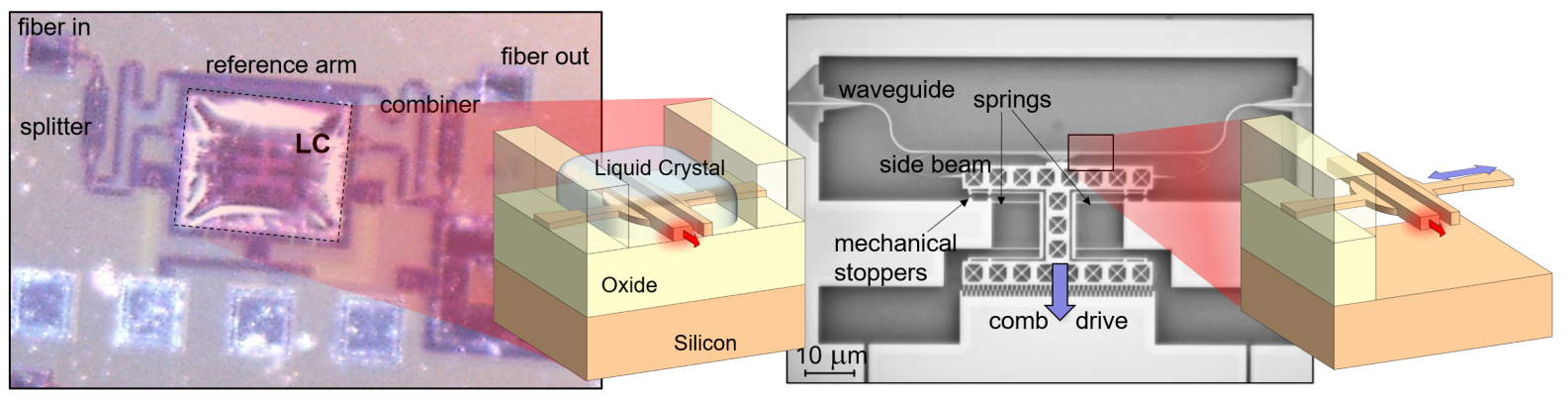

Fig. 2. Experimental demonstration of a phase shifter based on liquid-crystals [6] and MEMS [7].

\section{Heaters}

Heaters tune the refractive index of the silicon waveguide through the strong thermo-optic effect. The efficiency of the thermo-optic phase shifter is mostly determined by the heated volume and the thermal leakage rate. The power for a $\pi$ phase shift ranges from $30 \mathrm{~mW}$ down to $3 \mathrm{~mW}$ [2]. Heaters can be implemented using resistors in metal or doped silicon. With doped heaters it is possible to include a diode, enabling electrical multiplexing [5].

\section{Liquid Crystals}

Liquid crystals are birefringent materials that can be reoriented with an electric field. When used as a waveguide cladding, they can tune the effective index of the guided mode [3]. The driving field can be applied by top electrodes [3] or by electrodes next to the waveguide [6]. This lateral approach is easier to integrate in a silicon photonics waveguide platform as there is no constraint on the material stack of the back-end metallization: this stack can be opened locally, as shown in Fig. 2 With this approach, we achieved a phase shift of $0.8 \pi$ in $50 \mu \mathrm{m}$.

\section{MEMS}

A similar actuation geometry can be used to implement MEMS-based phase shifters. In this case, a side beam of silicon can be moved into the proximity of the waveguide core, thus affecting the evanescent field [7]. By using electrostatic actuation to move the silicon, a large phase shift can be achieved with very low electrical power consumption. The device illustrated in Fig. 2 achieves 1-2.5 $\pi$ phase shift in a $50 \mu \mathrm{m}$ length.

\section{Summary}

Large-scale photonic circuits require low-power, compact and low-loss electro-optic phase shifters. While heaters are flexible and still continuously improving, new techniques are are being introduced. We discuss our progress on liquid crystals and MEMS phase shifters, especially targeted at large-scale programmable photonic integrated circuits.

\section{Acknowledgements}

This work was supported by the European Union grants 780283 (MORPHIC) and 725555 (PhotonicSWARM).

\section{References}

1. W. Bogaerts et al., "Programmable photonic circuits," Nature 586, 207-216 (2020).

2. A. Masood et al., "Comparison of heater architectures for thermal control of silicon photonic circuits," in Proceedings of the 10th International Conference on Group IV Photonics, (2013), p. ThC2.

3. W. De Cort et al., "Wide tuning of silicon-on-insulator ring resonators with a liquid crystal cladding," Opt. Lett. 36, 3876-3878 (2011).

4. C. Errando-Herranz et al., "MEMS for Photonic Integrated Circuits," IEEE J. Sel. Top. Quantum Electron. 26, 1-1 (2019).

5. A. Ribeiro et al., "Column-Row Addressing of Thermo-Optic Phase Shifters for Controlling Large Silicon Photonic Circuits,” IEEE J. Sel. Top. Quantum Electron. 26, 1-8 (2020).

6. L. Van Iseghem et al., "Liquid crystal phase shifter integrated in a silicon photonics platform," in European Conference on Integrated Optics (ECIO), (2020), p. 1.3.

7. P. Edinger et al., "Compact low loss MEMS phase shifters for scalable field-programmable silicon photonics," in Conference on Lasers and Electro-Optics, (OSA, Washington, D.C., 2020), p. SM3J.2. 